\title{
Caputo Time Fractional Model Based on Generalized Fourier's and Fick's Laws for Jeffrey Nanofluid: Applications in Automobiles
}

\author{
Jawad Ahmad, ${ }^{1}$ Farhad Ali $\mathbb{D}^{1},{ }^{1}$ Saqib Murtaza $\mathbb{D}^{2},{ }^{2,3}$ and Ilyas Khan ${ }^{4}$ \\ ${ }^{1}$ Department of Mathematics, City University of Science and Information Technology, Peshawar, Khyber Pakhtunkhwa, Pakistan \\ ${ }^{2}$ Computational Analysis Research Group, Ton Duc Thang University, Ho Chi Minh City, Vietnam \\ ${ }^{3}$ Faculty of Mathematics and Statistics, Ton Duc Thang University, Ho Chi Minh City, Vietnam \\ ${ }^{4}$ Department of Mathematics, College of Science Al-Zulf, Majmah University, Al-Majmah 11952, Saudi Arabia
}

Correspondence should be addressed to Saqib Murtaza; saqibali6997@gmail.com

Received 16 April 2021; Accepted 11 August 2021; Published 21 August 2021

Academic Editor: Constantin Fetecau

Copyright $\odot 2021$ Jawad Ahmad et al. This is an open access article distributed under the Creative Commons Attribution License, which permits unrestricted use, distribution, and reproduction in any medium, provided the original work is properly cited.

\begin{abstract}
This article aims to examine Jeffery nanofluid with joint effects of mass and heat transfer in a horizontal channel. The classical model is transferred to the Caputo fractional model by using the generalized Fourier's and Fick's laws. The nanofluids are formed by dispersing two different nanoparticles, silver and copper, into a based fluid. A novel transformation has been applied to the mass and energy equation and then solved by using the sine Fourier and the Laplace transformation jointly. The exact solution is given in terms of a special function, that is, the Mittag-Leffler function. The Sherwood number and Nusselt number are calculated and displayed in the tabular form. The effect of embedded parameters on the velocity, concentration, and temperature profile is discussed graphically. It is noted that the heat transfer rate of EO is improved by $28.24 \%$ when the volume fraction of $\mathrm{Ag}$ nanoparticles is raised from 0.00 to 0.04 .
\end{abstract}

\section{Introduction}

The study of non-Newtonian fluid is very significant because it has vast applications in many areas, especially in the engineering and industrial sectors [1-4]. One of the wellknown models of non-Newtonian fluid is the Jeffrey fluid model which has both the property of viscosity and elasticity, and therefore it comes in the class of viscoelastic fluid. Engine oil, castor oil, and polymers are few examples of Jeffrey fluid. The Jeffrey fluid model has the facility to customize the time derivative on behalf of the convective derivative. The viscous fluid and second-grade fluid models can be recovered by taking Jeffrey fluid parameters equal to zero. Due to the above-stated applications, many scholars considered the Jeffrey model. Hayat et al. [5] studied the incompressible flow of viscoelastic Jeffrey fluid on a stretching sheet. For the solution of velocity and temperature field, the authors used the Homotopy method. The heat transfer phenomena of viscoelastic Jeffrey fluid at the stagnation point were discussed by Turkyilmazoglu and Pop
[6]. Ellahi and Hussain [7] observed the instantaneous behavior of partial slip and MHD effects on the wave-like flow of Jeffrey fluid. The separation of variables technique is used to get the closed-form solutions. Mabood et al. [8] examined the two-dimensional steady incompressible flow of Jeffrey fluid over a stretching sheet. To get solutions, the Runge-Kutta order four method has been applied. Moreover, Qasim [9] studied the Jeffrey fluid with mass and heat transfer in the occurrence of a heat source/heat sink. To get the exact solution, the author used the power series method. Two-phase dusty non-Newtonian fluid flow together with the impact of free convection and power law has been examined on a vertical surface by Siddiqa et al [10].

Cooling and lubricity are significant in several industries, especially in transportation and energy production. However, conventional fluids such as ethylene glycol, water, and oil have very low thermal conductivity, which is the main problem to innovation in thermal management and energy efficiency. To avoid the intrinsic limitation of convectional heat transfer fluid, a new thought of nanofluid was 
introduced by Choi and Eastman [11]. Due to various applications, many researchers used different nanoparticles to improve the base fluid's thermal and mechanical properties. Ali et al. [12] examined the efficiency of EO in the response of silver nanoparticles together with the effect of diffusionthermo in a heated rotatory system. Moreover, Kole and Dey [13] studied the influence of gear oil and dispersed copper oxide nanoparticles. They observed that nanofluid's viscosity is enhanced 3 times of the base fluid with the volume fraction of 0.025 . Tesfai et al. [14] empirically examined the application of controlling thermal systems and used graphene oxide and graphene nanoparticles. Sheikholeslami and Rokni [15] analyzed the simulation of the nanofluid heat transfer under the impact of magnetic field. The effects and significance of thermophoresis and Brownian motion in natural convection flow of nanofluid have been examined by Haddead et al. [16], Parekh and Lee [17], Dinvarad et al. [18], Mohyuddin et al. [19], Loganath [20], and Ferrouillat et al. [21]. They observed that nanoparticles are responsible for enhancing the thermal and mechanical properties of the base fluids. They also detected that nanofluids are more stable and do not have a sedimentation problem.

According to Eric Temple Bell [22], "Calculus is considered the most powerful tool" for scientific thought. Fractional calculus is a calculus with a noninteger order derivative. This was originated from a letter written by Leibniz in 1695 to Marquis de L'Hospital [23]. Later on, many mathematicians have been attracted and started work on this new topic. Euler, Riemann, Liouville, Laplace, Grunewald, Letniker, and so on worked on the fractional calculus. In the $18^{\text {th }}$ century, the Riemann-Liouville definition [24] of fractional derivative was mostly used definition. The deficiency that occurred in the Riemann-Liouville definition was removed by Caputo and presented a novel definition for fractional derivative [25]. However, it still contains the problem of singularity. The problem of singularity was fixed by Caputo-Fabrizio by giving a novel definition based on exponential function [26]. Nowadays, the implementation of fractional calculus is not limited to mathematics problems only but also contributes to solving the problems in many sectors like elasticity, chaos, diffusion, and polymerization. Fractional calculus is a very effective and efficient tool for elaborating heredity and the memory effect of the phenomena. In the last few years, remarkable development has been done using fractional calculus [27-30]. Bearing in mind the above-stated significance, many researchers contribute their potentials in the area of fractional calculus. Alizadeh et al. [31] discussed the transient response of the parallel circuit with the nonlocal derivative of the Caputo-Fabrizio. The authors used the Laplace transformed technique for their analysis. For the examination of the ground water pollution, Atangana and Alqahtani [32] used the time-space model of the Caputo-Fabrizio fractional derivative. Dokuyucu et al. [33] used the fractional derivative for the investigation of the tumor dealing model. Atangana and Alqahtani [32] discussed the ground level water problem using CF fractional derivative with the local and nonsingular kernel. Moreover, Ahmad et al. [34] discussed the existence and uniqueness of $\varphi$-Caputo pantograph differential equation. Keeping in view the applications of Caputo-Fabrizio fractional derivative, Doungmo Goufo [35] used the CF derivative for the analysis of Kortweg-de Vries-Burgers equation. With the help of CF derivative, Hristov [36] discussed the transient heat diffusion equation together with impact of nonsingular kernel fading memory. The author used the Cattaneo constitutive equation with Jeffrey's kernel. Koca and Atangana [37] utilized the nonlocal fractional derivative operator of Caputo-Fabrizio for the inspection of the Cattaneo-Hristov model together with the effect of elastic heat diffusion.

The present paper investigates the MHD flow of Jeffrey nanofluid in a horizontal channel with mass and heat transfer. Unlike the published work, the constitutive equations are transformed to fractional model by using generalized Fick's and Fourier's laws. A novel transformation has been applied to the energy and concentration equation and then solved by using the sine Fourier and the Laplace transformation jointly. The obtained general solutions satisfy all the conditions, which show the validity of the obtained general solutions.

\section{Mathematical Modeling}

We considered the laminar and unsteady flow of Jeffrey nanofluid in a horizontal channel in which both the plates are separated by distance $l$. The flow is assumed to be in the direction of $x$-axis with ambient temperature $T_{a}$ and ambient concentration $C_{a}$. Initially, both the plates and fluid are at rest. At the time $t=0^{+}$, the upper plate temperature and concentration levels are increased to $T_{a}+\left(T_{p}-T_{a}\right) A(t)$ and $C_{a}+\left(C_{p}-C_{a}\right) B(t)$, respectively. The physical geometry of the problem is given in Figure 1 .

The constitutive equations which govern the flow using Boussinesq's approximation $[38,39]$ are given by

$$
\begin{aligned}
\rho_{n f} \frac{\partial u(y, t)}{\partial t}= & \frac{\mu_{n f}}{1+\lambda_{1}}\left(1+\lambda_{2} \frac{\partial}{\partial t}\right) \frac{\partial^{2} u}{\partial y^{2}}-\sigma_{n f} B_{0}^{2} u(y, t)+\left(\rho \beta_{T}\right)_{1 n f} \mathcal{g}_{1}\left(T-T_{a}\right) \\
& +\left(\rho \beta_{c}\right)_{1 n f} \mathcal{g}_{1}\left(C-C_{a}\right) \\
\left(\rho C_{p}\right)_{n f} \frac{\partial T_{1}(y, t)}{\partial t}= & -\frac{\partial q(y, t)}{\partial y}
\end{aligned}
$$




$$
\begin{aligned}
q(y, t) & =-k_{n f} \frac{\partial T_{1}(y, t)}{\partial y}, \\
\frac{\partial C(y, t)}{\partial t} & =-\frac{\partial \gamma_{1}(y, t)}{\partial y}, \\
\gamma(y, t) & =-D_{n f} \frac{\partial C(y, t)}{\partial y}
\end{aligned}
$$

with physical condition as follows:

$$
\left.\begin{array}{lll}
u(y, 0)=0, & T(y, 0)=0, & C(y, 0)=0, \\
u(0, t)=0, & T(0, t)=T_{a}, & C(0, t)=C_{a}, \\
u(l, t)=0, & T(l, t)=T_{a}+\left(T_{p}-T_{a}\right) A(t), & C(l, t)=C_{a}+\left(C_{p}-C_{a}\right) B(t) .
\end{array}\right\}
$$

The following dimensionless variables are used for dimensional analysis:

$$
\begin{aligned}
u_{1} & =\frac{u}{U_{0}}, \\
\xi & =\frac{y}{l}, \\
\tau & =\frac{v}{l^{2}} t, \\
\theta & =\frac{T_{1}-T_{1 a}}{T_{p}-T_{a}} \\
\phi & =\frac{C_{1}-C_{1 a}}{C_{p}-C_{a}} \\
\lambda & =\frac{q l}{k_{n f}\left(T_{p}-T_{a}\right)}, \\
\delta & =\frac{\gamma l}{D_{n f}\left(C_{p}-C_{a}\right)}, \\
B(\tau) & =B\left(\frac{l^{2}}{v} t\right) . \\
A(\tau) & =A\left(\frac{l^{2}}{v} t\right),
\end{aligned}
$$

Using above-stated dimensionless variables, equations (1)-(6) become

$$
\begin{aligned}
\frac{\partial u_{1}}{\partial \tau} & =\frac{m_{0}}{1+\lambda_{1}}\left(1+\lambda_{3} \frac{\partial}{\partial \tau}\right) \frac{\partial^{2} u_{1}}{\partial \xi^{2}}-M_{0} u_{1}+G r_{0} \theta+G m_{0} \phi \\
\frac{\partial \theta}{\partial \tau} & =\frac{1}{a_{0}} \frac{\partial \lambda(\xi, t)}{\partial \xi}
\end{aligned}
$$

$$
\lambda(\xi, t)=-\frac{\partial \theta}{\partial \xi}
$$

$u_{1}(\xi, 0)=0, \quad \theta(\xi, 0)=0, \quad \phi(\xi, 0)=0$,

$u_{1}(0, \tau)=0, \quad \theta(0, \tau)=0, \quad \phi(0, \tau)=C_{a}$,

$u(1, \tau)=0, \quad \theta(1, \tau)=A(\tau), \quad \phi(1, \tau)=B(t)$.

The following are the dimensionless parameters and some constants after the dimensionalization process:

$$
\begin{aligned}
G r & =\frac{g l^{2} \beta_{T}\left(T_{p}-T_{a}\right)}{U_{0} \nu} b_{0}, \\
G_{r 0} & =\frac{G r}{\varphi_{0}}, \\
M & =\frac{\delta B_{0}{ }^{2} l^{2}}{\mu}, \\
G m & =\frac{g \beta_{c} l^{2}\left(C_{p}-C_{a}\right)}{U_{0} v}, \\
S c & =\frac{\nu}{D_{n f}}, \\
M_{0} & =\frac{M}{\varphi_{0}},
\end{aligned}
$$$$
G m_{o}=\frac{G m}{\varphi_{0}},
$$ 


$$
\begin{aligned}
& \lambda_{3}=\frac{\lambda_{2} v}{l^{2}}, \\
& a_{o}=\frac{\varphi_{1} p r}{\varphi_{2}}, \\
& p r=\frac{v \rho c_{p}}{k_{f}}, \\
& b_{0}=\frac{S c}{1-\varphi}, \\
& \phi_{0}=(1-\varphi)+\varphi \frac{\rho_{s}}{\rho_{f}}, \\
& m_{0}=\left((1-\varphi)^{2.5}\left(1-\varphi+\varphi \frac{\rho_{s}}{\rho_{f}}\right)\right)^{-1}, \\
& \phi_{1}=1-\varphi+\varphi \frac{\left(\rho c_{p}\right)_{s}}{\left(\rho c_{p}\right)_{f}} \\
& \phi_{2}=\frac{k_{s}-2 k_{f}-2 \varphi\left(k_{f}-k_{s}\right)}{k_{s}+2 k_{f}+\varphi\left(k_{f}-k_{s}\right)} .
\end{aligned}
$$

A fractional model is developed using the generalized Fick's and Fourier laws as follows:

$$
\begin{array}{ll}
\lambda(\xi, \tau)=-{ }^{c} \wp_{\tau}^{1-\alpha}\left(\frac{\partial \theta(\xi, \tau)}{\partial \xi}\right), & 0<\alpha \leq 1, \\
\delta(\xi, \tau)=-{ }^{c} \wp_{\tau}^{1-\alpha}\left(\frac{\partial \phi(\xi, \tau)}{\partial \xi}\right), & 0<\alpha \leq 1,
\end{array}
$$

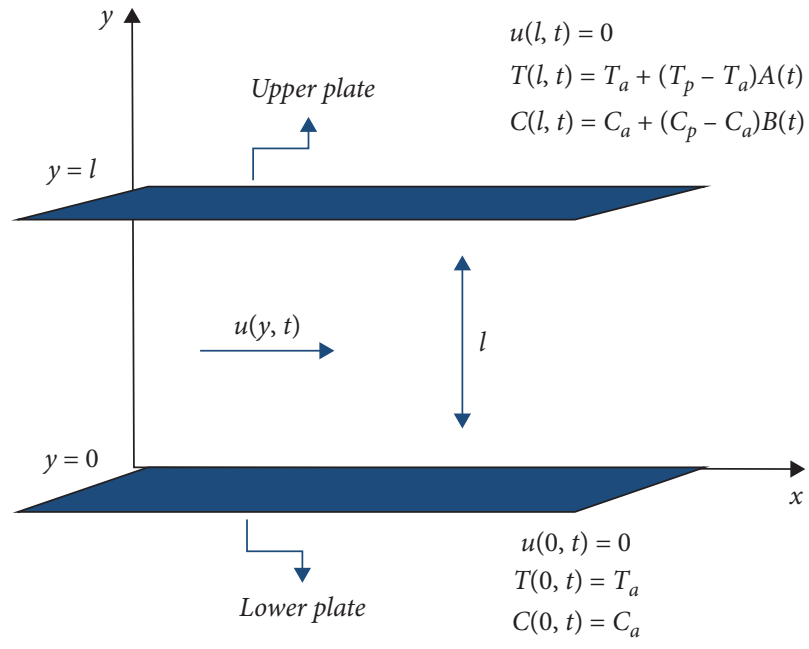

FIGURE 1: Flow regime of problem.

TABle 1: Thermo-mechanical properties of the base fluid and nanoparticles [12, 40].

\begin{tabular}{lccccc}
\hline Properties & $\rho$ & $C_{p}$ & $k$ & $\beta$ & $\operatorname{Pr}$ \\
\hline Engine oil & 863 & 2048 & 0.1404 & 0.00007 & 6000 \\
$\mathrm{Cu}$ & 8933 & 385 & 401 & $1.67 \times 10^{-5}$ & - \\
$\mathrm{Ag}$ & 10500 & 235 & 429 & $1.89 \times 10^{-5}$ & - \\
\hline
\end{tabular}

where ${ }^{c} \wp_{\tau}^{\alpha}($.$) is the Caputo time operative which is as follows:$

$$
{ }^{c} \wp_{\tau}^{\alpha} f(y, t)=\frac{1}{\Gamma(1-\alpha)} \int_{0}^{t_{1}} f_{1}(y, s)\left(t_{1}-s_{1}\right)^{-\alpha_{1}} \mathrm{~d} s=\eta_{\alpha 1}\left(t_{1}\right) * f\left(y, t_{1}\right), \quad 0<\alpha \leq 1,
$$

where $\eta_{\alpha}(t)=\left(t^{-\alpha} / \Gamma(1-\alpha)\right)$ is the kernel of the singular power law.

Utilizing the definition of Caputo time derivative, using equations (9), (11), (15), and (17), we arrived at

$$
\begin{gathered}
\frac{\partial \theta(\xi, \tau)}{\partial t}=\frac{1}{a_{0}}{ }^{c} \wp_{\tau}^{1-\alpha}\left(\frac{\partial^{2} \theta(\xi, \tau)}{\partial \xi^{2}}\right), \\
\frac{\partial \phi(\xi, \tau)}{\partial t}=\frac{1}{b_{0}}{ }^{c} \wp_{\tau}^{1-\alpha}\left(\frac{\partial^{2} \phi(\xi, \tau)}{\partial \xi^{2}}\right) .
\end{gathered}
$$

Equations (18) and (19) can be written as follows:

$$
\begin{aligned}
& \wp_{\tau}^{\alpha} \theta(\xi, \tau)=\frac{1^{c}}{a_{0}} \frac{\partial^{2} \theta(\xi, \tau)}{\partial \xi^{2}}, \\
& \wp_{\tau}^{\alpha} \phi(\xi, \tau)=\frac{1}{b_{0}} \frac{\partial^{2} \phi(\xi, \tau)}{\partial \xi^{2}} .
\end{aligned}
$$

The thermo-mechanical properties of the considered base fluid and solid nanoparticles are given in Table 1.

2.1. Solution of Energy Equation. Using the following transformation:

$$
\mathrm{X}(\xi, \tau)=\theta(\xi, \tau)-\xi A(\tau),
$$

on equation (20), we get

$$
{ }^{c} \wp_{\tau}^{1-\alpha} \mathrm{X}(\xi, \tau)+\xi^{c} \wp_{\tau}^{1-\alpha} A(\tau)=\frac{1}{a_{0}} \frac{\partial^{2} \mathrm{X}(\xi, \tau)}{\partial \xi^{2}},
$$

according to equation (22), and the initial and boundary conditions are as follows:

$$
\begin{aligned}
& X(\xi, 0)=0, \\
& X(0, \tau)=0, \\
& X(1, \tau)=0
\end{aligned}
$$


Taking the Laplace transformation on equation (23), we get

$$
s^{\alpha} \overline{\mathrm{X}}(\xi, s)+\xi s^{\alpha} \bar{A}(s)=\frac{1}{a_{0}} \frac{d^{2} \overline{\mathrm{X}}(\xi, s)}{d \xi^{2}} .
$$

Now, applying sine Fourier transform to equation (25), we get

$$
s^{\alpha} \overline{\mathrm{X}}_{F 1}\left(n_{1}, s\right)-\frac{(-1)^{n}}{n \pi} s^{\alpha} \bar{A}(s)=-\frac{1}{a_{0}} \overline{\mathrm{X}}_{F}\left(n_{1}, s\right)\left(n_{1} \pi\right)^{2} .
$$

After simplification, we arrived at

$$
\overline{\mathrm{X}}_{F 1}\left(n_{1}, s\right)=s \bar{A}(s) \frac{(-1)^{n}}{n_{1} \pi} \frac{s^{\alpha-1}}{s^{\alpha}+\left(\left(n_{1} \pi\right)^{2} / a_{0}\right)} \text {. }
$$

Inverting the essential transformation of equation (26), we get

$$
\mathrm{X}(\xi, \tau)=2 \sum_{n=1}^{\infty} \frac{(-1) \sin (\xi n \pi)}{n \pi} \int_{0}^{\tau} A(\tau-t) E_{\alpha, \alpha+1}\left(-\frac{(n \pi)^{2}}{a_{0}} t^{\alpha}\right) \mathrm{d} t
$$

Consequently, the exact solution of equation (20) is as follows:

$$
\theta(\xi \cdot \tau)=\mathrm{X}(\xi, \tau)+\xi A(\tau)
$$

2.2. The Solution of Mass Equation. Using the following transformation:

$$
\mathfrak{R}(\xi, \tau)=\phi(\xi, \tau)-\xi B(\tau),
$$

on equation (21), we get

$$
{ }^{c} \wp_{\tau}^{1-\alpha} \mathfrak{R}(\xi, \tau)+\xi^{c} \wp_{\tau}^{1-\alpha} B(\tau)=\frac{1}{b_{0}} \frac{\partial^{2} \mathfrak{R}(\xi, \tau)}{\partial \xi^{2}} .
$$

According to equation (30), the transformed conditions are as follows:

$$
\begin{aligned}
& \Re(\xi, 0)=0, \\
& \Re(0, \tau)=0, \\
& \Re(1, \tau)=0 .
\end{aligned}
$$

Taking the Laplace on both sides of equation (30), we get

$$
s^{\alpha} \bar{\Re}(\xi, s)+\xi s^{\alpha} \bar{B}(s)=\frac{1}{b_{0}} \frac{d^{2} \bar{\Re}(\xi, s)}{d \xi^{2}} .
$$

Now, taking sine Fourier transform to equation (33), we get

$$
s^{\alpha} \overline{\mathfrak{R}}_{F}(n, s)-\frac{(-1)^{n}}{n \pi} s^{\alpha} \bar{B}(s)=-\frac{1}{b_{0}} \overline{\mathfrak{R}}_{F}(n, s)(n \pi)^{2},
$$

and after simplification, we arrived at

$$
\overline{\mathfrak{R}}_{F}(n, s)=s \bar{B}(s) \frac{(-1)^{n}}{n \pi} \frac{s^{\alpha-1}}{s^{\alpha}+\left((n \pi)^{2} / b_{0}\right)} .
$$

Inverting the essential transformation of equation (35), we get

$$
\Re(\xi, \tau)=2 \sum_{n=1}^{\infty} \frac{(-1) \sin (\xi n \pi)}{n \pi} \int_{0}^{\tau} B(\tau-t) E_{\alpha, \alpha+1}\left(-\frac{(n \pi)^{2}}{b_{0}} t^{\alpha}\right) \mathrm{d} t .
$$

Consequently, the final exact solution of equation (21) is as follows:

$$
\phi(\xi, \tau)=\Re(\xi, \tau)+\xi B(\tau)
$$

2.3. Solution of the Velocity Distribution. Taking the Laplace of equation (8), we get

$$
s u_{1}(\xi, s)=\frac{m_{0}}{1+\lambda_{1}}\left(1+\lambda_{3} s\right) \frac{d^{2} \bar{u}_{1}}{d \xi^{2}}-M_{0} \bar{u}_{1}+G r_{0} \bar{\theta}+G m_{0} \bar{\phi}
$$

Applying sine Fourier transform to equation (36) and after simplification, we obtained the following:

$$
u_{1 F}(n, s)=\frac{G r_{0}\left(1+\lambda_{1}\right)}{\left(s+A_{0}\right)\left(1+\lambda_{1}\right)+\lambda_{3} m_{0}(n \pi)^{2}} \bar{\theta}_{F}(n, s)+\frac{G m_{0}\left(1+\lambda_{1}\right)}{\left(s+A_{0}\right)\left(1+\lambda_{1}\right)+\lambda_{3} m_{0}(n \pi)^{2}} \bar{\phi}_{F}(n, s) \text {, }
$$

where $A_{0}=\left(m_{0}(n \pi)^{2}+M_{0}\left(1+\lambda_{1}\right) /\left(1+\lambda_{1}\right)+\lambda_{3} m_{0}(n \pi)^{2}\right)$; incorporating the value of $\bar{\theta}_{F}(n, s)$ and $\bar{\phi}_{F}(n, s)$ in equation (39) and taking the Laplace inverse, we get 


$$
\begin{aligned}
u_{1 F}(n, \tau)= & G r_{0} A_{1}\left(\frac{(-1)^{n}}{n \pi}\right) \exp \left(A_{0} \tau\right) *\left(\int_{0}^{\tau} A(\tau-t) E_{\alpha, \alpha-1}\left(\frac{-(n \pi)^{2}}{a_{0}} t^{\alpha}\right) \mathrm{d} t-A(\tau)\right) \\
& +G m_{0} A_{1}\left(\frac{(-1)^{n}}{n \pi}\right) \exp \left(A_{0} \tau\right) *\left(\int_{0}^{\tau} B(\tau-t) E_{\alpha, \alpha-1}\left(\frac{-(n \pi)^{2}}{a_{0}} t^{\alpha}\right) \mathrm{d} t-B(\tau)\right)
\end{aligned}
$$

where $A_{1}=\left(1+\lambda_{1} /\left(1+\lambda_{1}\right)+\lambda_{3} m_{0}(n \pi)^{2}\right)$.
Now, applying the inverse sine Fourier transform of equation (40),

$$
\begin{aligned}
u_{1}(\xi, \tau)= & 2 \sum_{n=1}^{\infty}\left\{G r_{0} A_{1}\left(\frac{(-1)^{n}}{n \pi}\right) \exp \left(A_{0} \tau\right) *\left(\int_{0}^{\tau} A(\tau-t) E_{\alpha, \alpha-1}\left(\frac{-(n \pi)^{2}}{a_{0}} t^{\alpha}\right) \mathrm{d} t-A(\tau)\right)\right\} \sin (n \pi \xi) \\
& +2 \sum_{n=1}^{\infty}\left\{G r_{0} A_{1}\left(\frac{(-1)^{n}}{n \pi}\right) \exp \left(A_{0} \tau\right) *\left(\int_{0}^{\tau} B(\tau-t) E_{\alpha, \alpha-1}\left(\frac{-(n \pi)^{2}}{a_{0}} t^{\alpha}\right) \mathrm{d} t-B(\tau)\right)\right\} \sin (n \pi \xi),
\end{aligned}
$$

where $E_{1 a, b}(\cdot)$ is the Mittag-Leffler function.

2.4. Skin Friction. For Jeffery nanofluid, skin friction is given by

$$
S_{f}=\left.\frac{1}{1+\lambda_{1}}\left(1+\lambda_{2} \frac{\partial}{\partial t}\right) \frac{\partial u_{1}}{\partial \xi}\right|_{\xi=0}
$$

2.5. Nusselt Number. For industrialists, the Nusselt number is a significant quantity, which is defined as follows:

$$
N_{n}=-\left.\frac{k_{n f}}{k_{f}} \frac{\partial \theta(\xi, \tau)}{\partial \xi}\right|_{\xi=1} .
$$

2.6. Sherwood Number. In nondimensional form, the Sherwood number is defined as follows:

$$
S_{n}=-\left.D_{n f} \frac{\partial \phi(\xi, \tau)}{\partial \xi}\right|_{\xi=1} .
$$

\section{Results and Discussion}

The incompressible flow of Jeffrey nanofluid in a bounded channel is elaborated in this article. The joint effect of mass and heat transfer has been studied. Fick's and Fourier's laws are used to develop the fractional model. To get the closedform solution, a novel technique is applied to change the equation into a simple form, and then Fourier's and Laplace transforms are used. The influence of embedded parameters on the velocity, temperature, and concentration distribution is presented in Figures 2-14.

Response of velocity profile against fractional order $\alpha$ is portrayed in Figure 2. In the classical order derivative, we have only one fluid layer for the analysis of considered fluid. The key feature of using fractional order derivative is to obtain more than one fluid layer for the investigation of the fluid rheology as shown in Figure 2. Because of this salient advantage, the experientialists can compare their results with one of the layer which will be best fitted to their solution. Figure 3 interprets the comparative examination of the copper and silver nanoparticles on velocity field. From the figure, the magnitude of the velocity for silver nanofluid is greater than the magnitude of the velocity of copper nanofluid. It is because that the density of silver nanoparticle is $\left(10497 \mathrm{~kg} / \mathrm{m}^{3}\right)$ and the density of copper is $\left(8940 \mathrm{~kg} / \mathrm{m}^{3}\right)$. Figures 4 and 5 represent the influence of generalized Jeffery fluid parameters $\lambda_{1}$ and $\lambda_{2}$ on the velocity distribution. It is clear from the figure that for greater values of $\lambda_{1}$, the velocity profile rises. This is because $\lambda_{1}$ is the time relaxation parameter, and due to the quick response of shear stresses, the fluid accelerates. In contrast, the greater value of $\lambda_{2}$ decreases the velocity distribution due to the delay response of shear stress. Figure 6 illustrates the impact of Gr (thermal Grashof number) on velocity distribution. If $\mathrm{Gr}$ rises, the velocity of the fluid also rises. Because of greater values of $\mathrm{Gr}$, the difference between the temperature of plate and surrounding temperature increases which leads to decrease in the viscous forces, and due to this, the motion of the fluid boosts up. Figure 7 describes the influence of velocity distribution for distinct values of $\mathrm{Gm}$ (mass Grashof number). It is noted from the sketch that rising values of $\mathrm{Gm}$ also boost up the fluid velocity. It is because of greater values of $\mathrm{Gm}$ the difference between the surrounding concentration and concentration on the plate increases which consequently enhances the fluid motion. Figure 8 shows that fluid velocity falls by rising the magnitude of Sc (Schmidt number). This is true because the Schmidt number has direct relation with the viscosity of the fluid. The behavior of the velocity distribution in response of volume fraction $\varphi$ is given Figure 9. It is noted from the sketch that velocity profile against $\varphi$ fell down. It is physically true because nanoparticles enhance the viscous powers in the fluid; due to this, it delays the motion. This outcome is of vital attentiveness. It is observed that hanging nanoparticles in engine oil improve the interconnected forces which can improve the life span and 


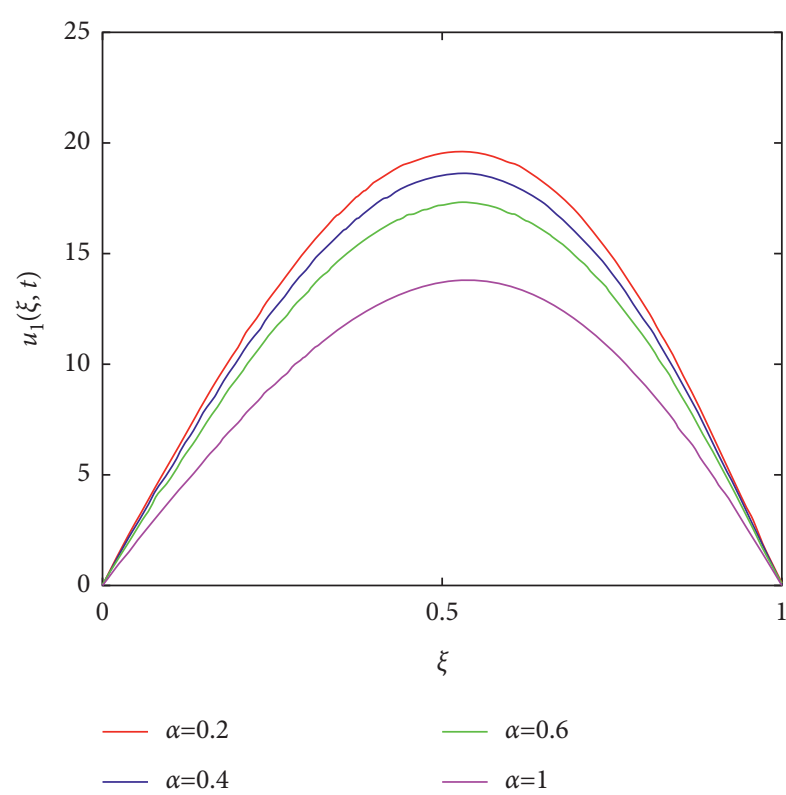

Figure 2: Deviation in velocity distribution for the distinct value $\alpha$.

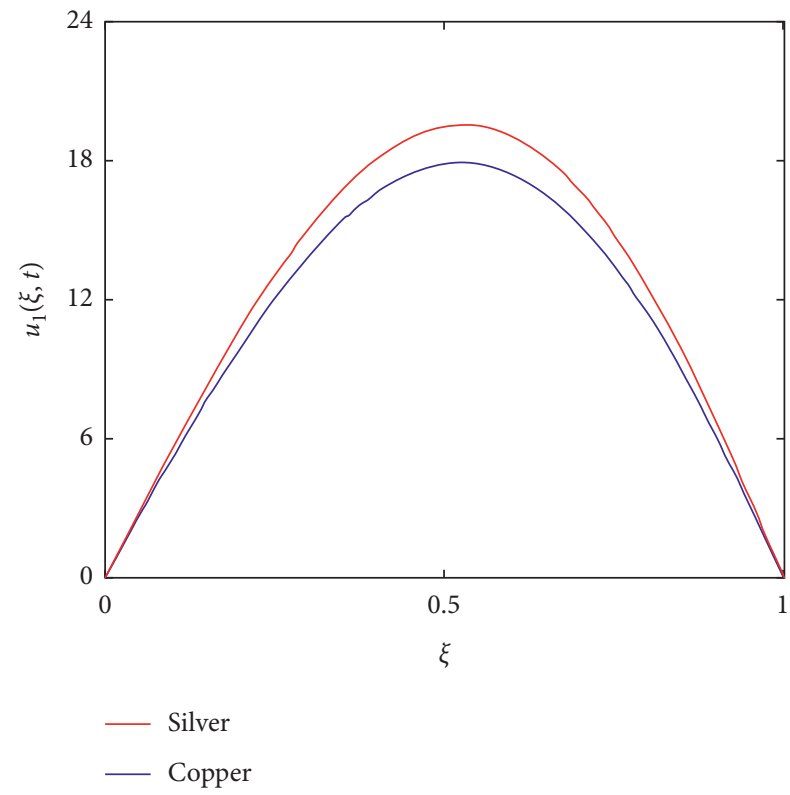

Figure 3: Comparison of silver and copper nanoparticles on the velocity profile.

thermo-mechanical properties of the engine oil. Figure 10 shows variation in the velocity profile of the Jeffrey fluid against $M$ (Hartman number. Higher values of $M$ generate drag forces (Lorentz) which lead to suppress the motion of the fluid. Figure 11 displays the behavior of temperature profile against the fractional order $\alpha$. This is the beauty of the fractional derivative because it gives more than one temperature profile for the investigation as we discussed in Figure 2. Figure 12 shows the comparison of silver and copper nanoparticles on temperature distribution. The figure displays that the silver nanoparticle has a higher temperature as compared with the copper nanoparticle. The

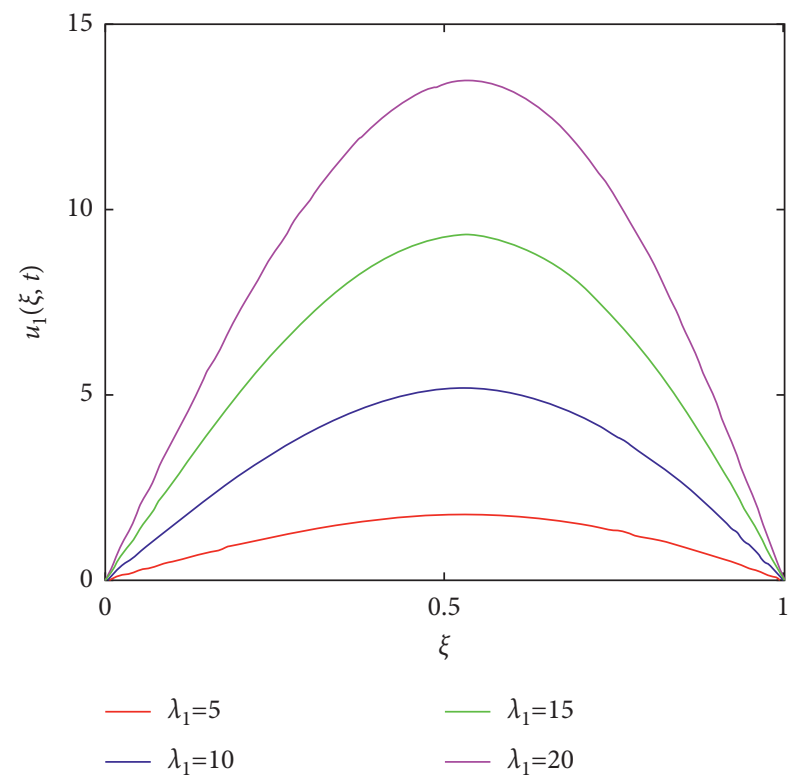

FIGURE 4: Outcome of time relaxation parameter $\lambda_{1}$ on the velocity distribution.

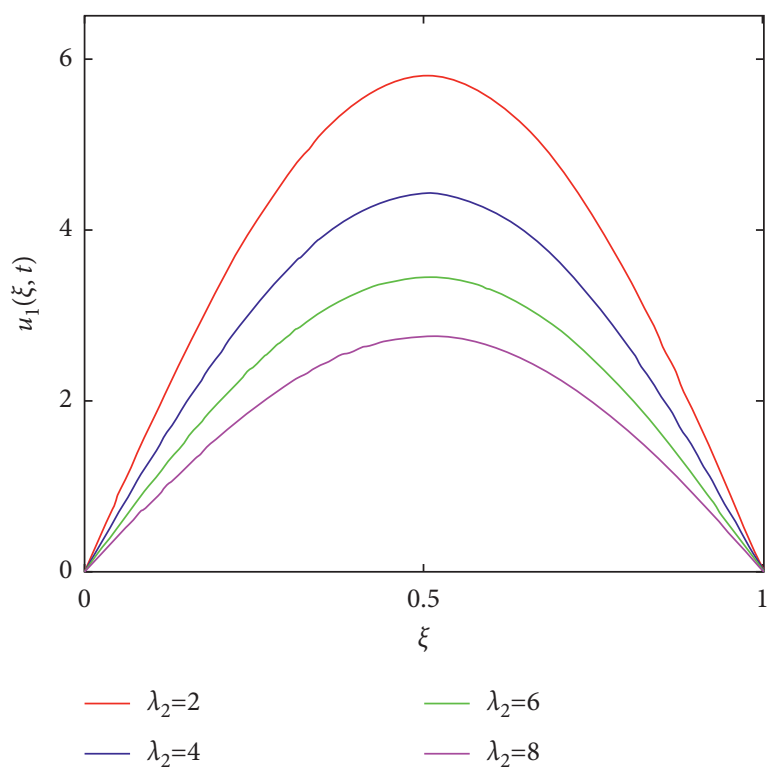

FIgURE 5: Impact of retardation time parameter $\lambda_{2}$ on the velocity distribution.

thermal conductivity of silver nanoparticle is $(406 \mathrm{~W} / \mathrm{m} \cdot \mathrm{K})$ and thermal conductivity of copper nanoparticle is $(385 \mathrm{~W} / \mathrm{m} \cdot \mathrm{K})$. Because of this difference, silver nanoparticle will conduct more temperature as compared with copper. Figure 13 reveals the performance of $\varphi$ (volume fraction) on temperature distribution. As for higher value of $\varphi$, the absorption ability of the fluid rises, and due to this, the fluid temperature is improved. Figure 14 highlights the influence of fractional order $\alpha$ on concentration distribution. The same trend is observed as in temperature and velocity profile. Decay in the concentration profile has been observed for rises $S c$ (Schmidt number) which is revealed in Figure 15. 


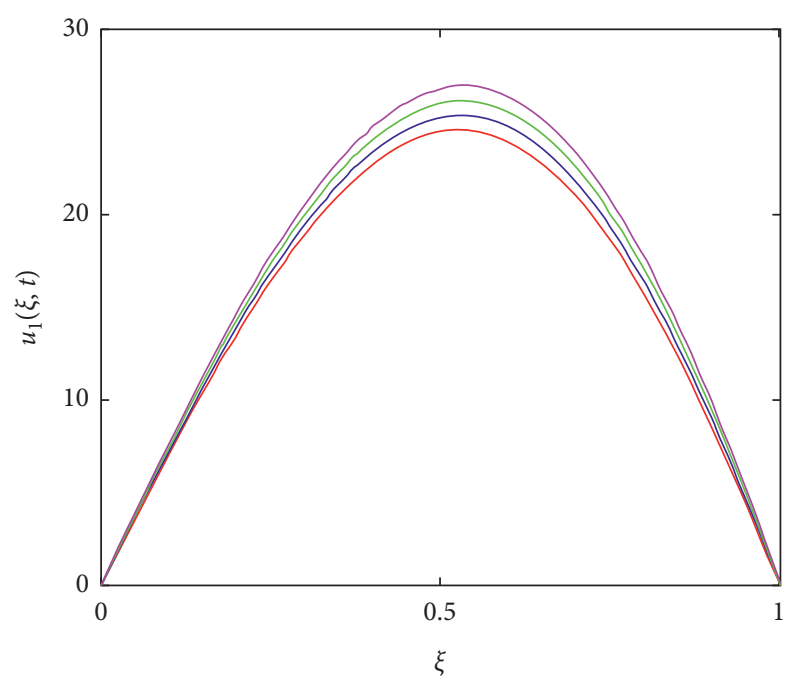
- Gr=5
- Gr $=15$
- Gr $=10$
- Gr $=20$

FIGURE 6: Impact of Gr on the velocity profile.

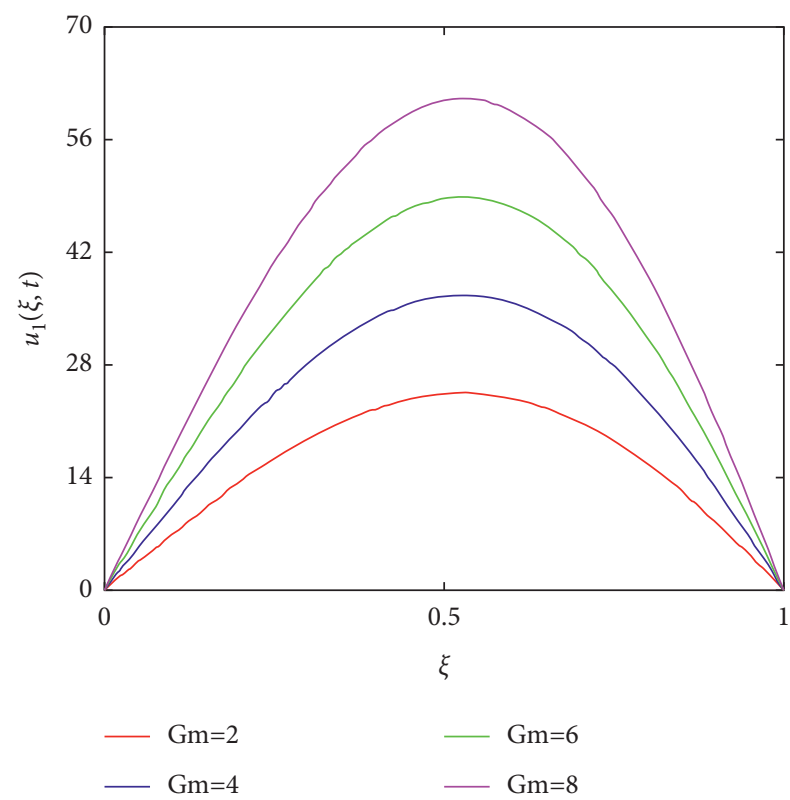

FIGURE 7: Effect of Gm on the velocity distribution.

Furthermore, Table 2 represents the variation of skin friction in the counter of corresponding nondimensional parameters. Skin friction is calculated numerically using MATHCAD software. Table 3 shows that the silver

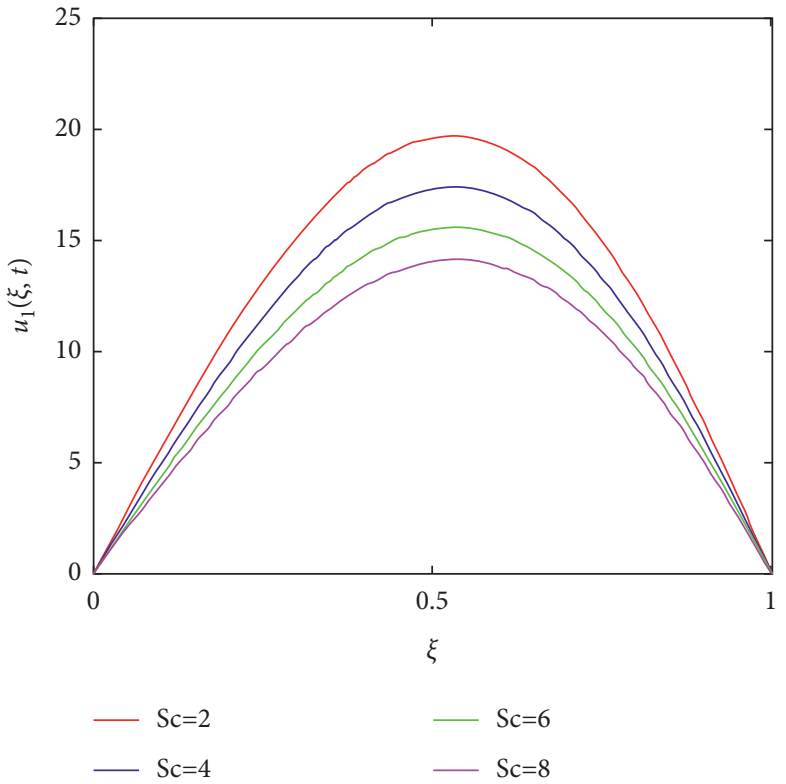

FIGURE 8: Influence of Sc on the velocity profile.

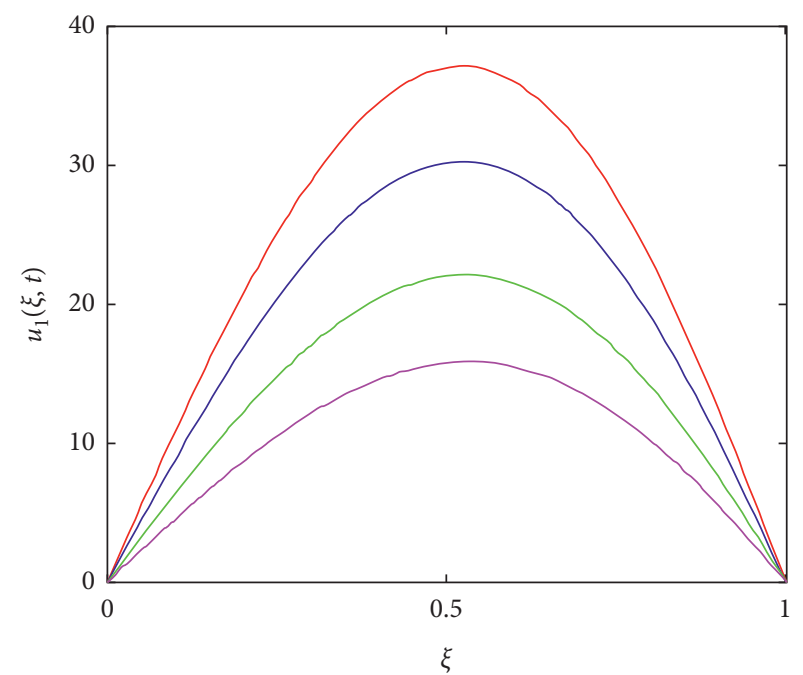

$$
\begin{aligned}
-\varphi=0.01 & -\varphi=0.03 \\
\varphi=0.02 & -\varphi=0.04
\end{aligned}
$$

FIGURE 9: Impact of volume fraction $\varphi$ on the velocity profile.

nanoparticles are better than the copper nanoparticles for enhancement of heat transfer rate in engine oil, while Table 4 shows the variation in Sherwood numbers. 


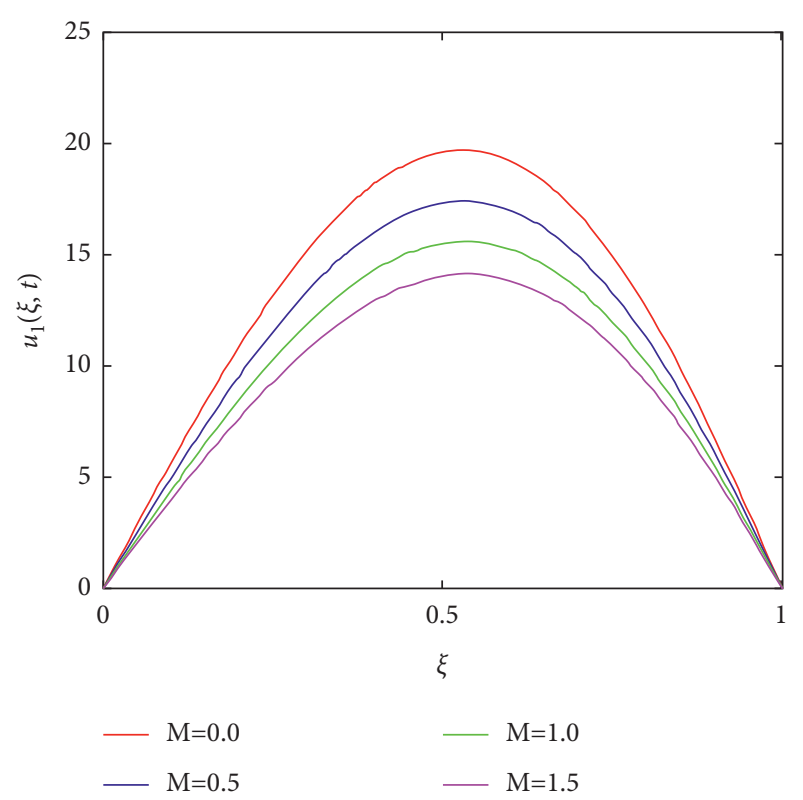

Figure 10: Impact of the Hartman number $M$ on the velocity profile.

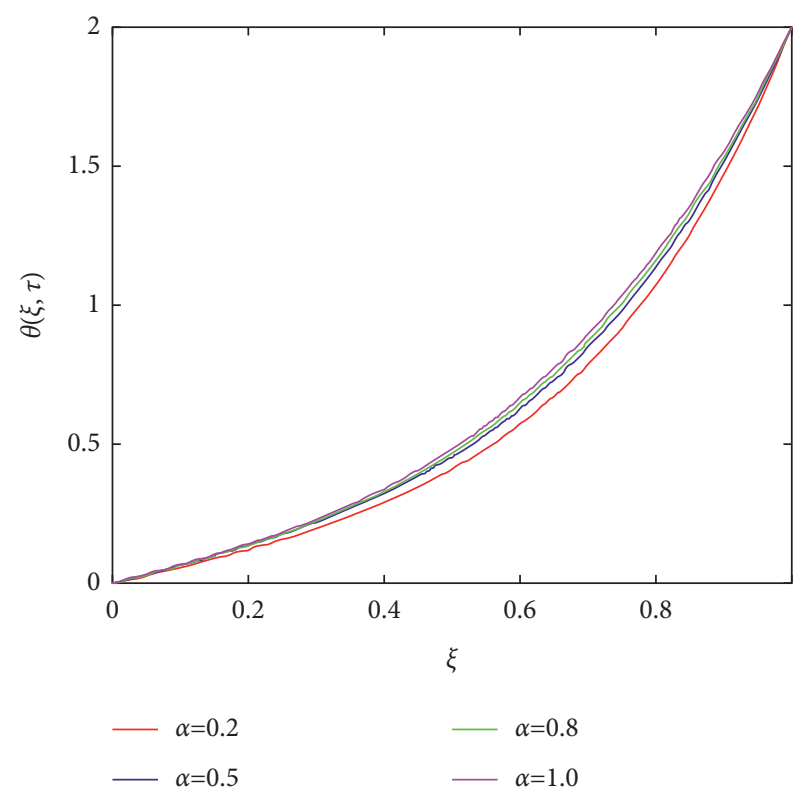

FIgURe 11: Deviation in temperature distribution for the distinct value of $\alpha$.

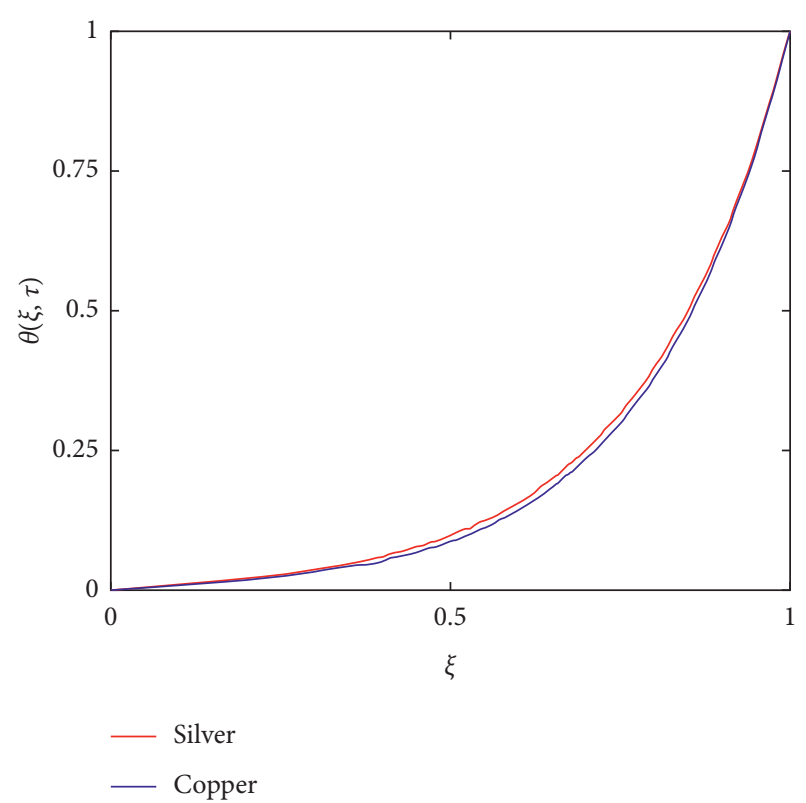

Figure 12: Evaluation of silver and copper nanoparticle on the temperature distribution.

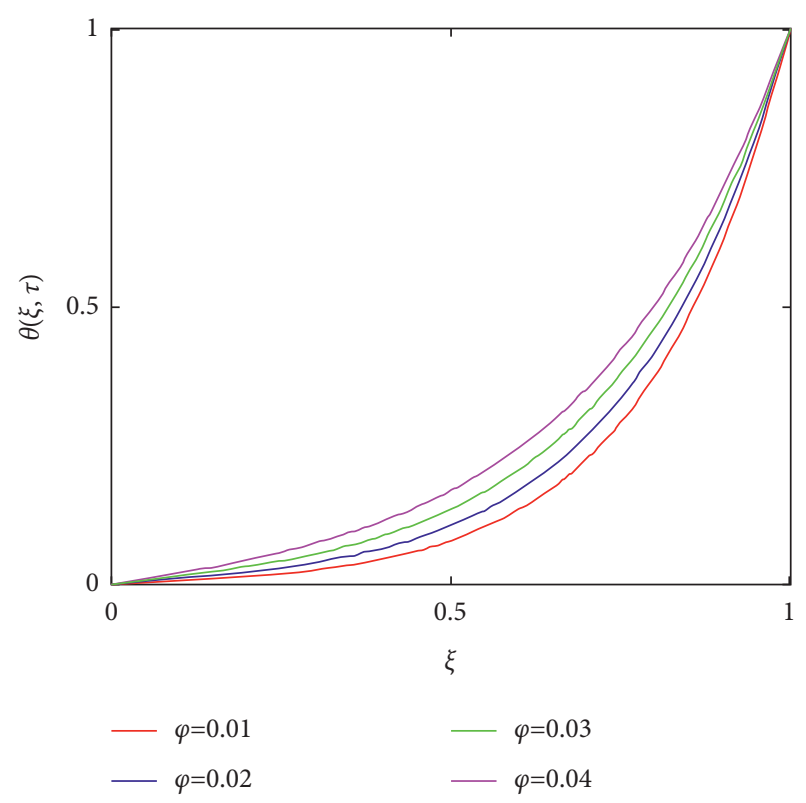

FIgURE 13: Influence of volume fraction $\varphi$ on the temperature distribution. 


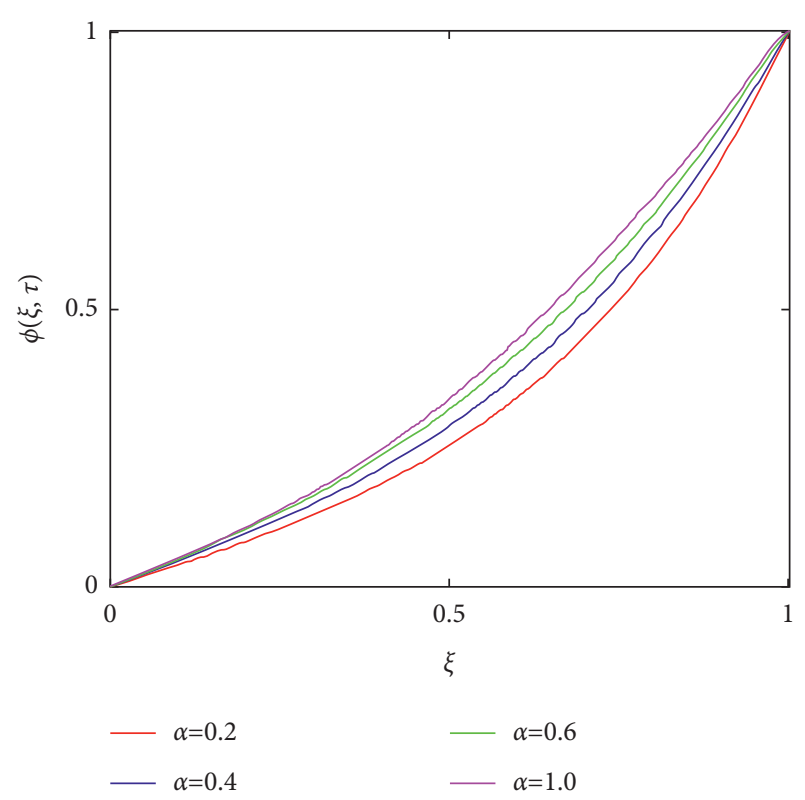

FIGURE 14: Variation in concentration profile for the distinct value of $\alpha$.

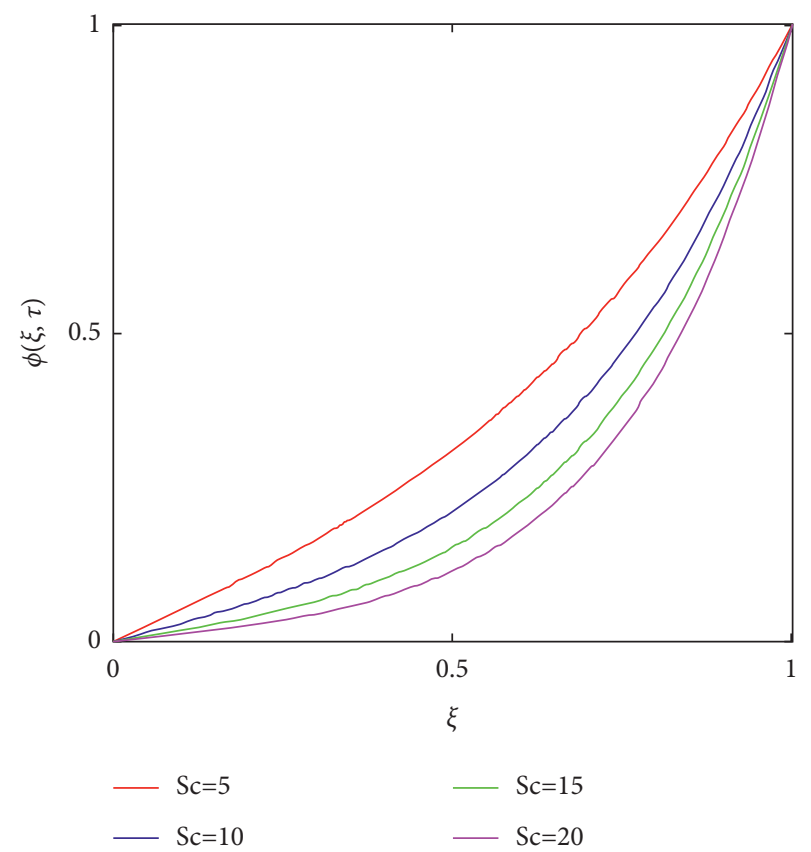

FIgURE 15: Influence of the Schmitt number Sc on the concentration profile.

\section{Conclusion Remarks}

In this paper, a novel technique is used to establish the fractionalize model of Jeffrey nanofluid. To fractionalize the model, the generalized Fourier and Fick's laws are used. To get the closed-form solution, a novel transformation has been used and then solved by the Fourier sine and the Laplace transform techniques. The acquired results are drawn and displayed in tables. By the above results and
TABLE. 2: Skin friction variation with corresponding embedded parameters.

\begin{tabular}{lcccccccc}
\hline$\alpha$ & $\mathrm{Gr}$ & $\mathrm{Gm}$ & $\mathrm{Sc}$ & $M$ & $\varphi$ & $\lambda_{1}$ & $\lambda_{2}$ & $S_{f}$ \\
\hline 0.2 & 5.0 & 5.0 & 5.0 & 0.5 & 0.01 & 0.5 & 0.5 & 1.915 \\
$\mathbf{0 . 3}$ & 5.0 & 5.0 & 5.0 & 0.5 & 0.01 & 0.5 & 0.5 & 1.940 \\
0.2 & $\mathbf{1 0}$ & 5.0 & 5.0 & 0.5 & 0.01 & 0.5 & 0.5 & 1.815 \\
0.2 & 5.0 & $\mathbf{1 0}$ & 5.0 & 0.5 & 0.01 & 0.5 & 0.5 & 1.805 \\
0.2 & 5.0 & 5.0 & $\mathbf{1 0}$ & 0.5 & 0.01 & 0.5 & 0.5 & 2.050 \\
0.2 & 5.0 & 5.0 & 5.0 & $\mathbf{1 . 0}$ & 0.01 & 0.5 & 0.5 & 2.150 \\
0.2 & 5.0 & 5.0 & 5.0 & 0.5 & $\mathbf{0 . 0 2}$ & 0.5 & 0.5 & 2.450 \\
0.2 & 5.0 & 5.0 & 5.0 & 0.5 & 0.01 & $\mathbf{1 . 0}$ & 0.5 & 2.501 \\
0.2 & 5.0 & 5.0 & 5.0 & 0.5 & 0.01 & 0.5 & $\mathbf{1 . 0}$ & 1.801 \\
\hline
\end{tabular}

TABLE. 3: Variation in the Nusselt number (rate of heat transfer).

\begin{tabular}{lcccccc}
\hline$\varphi$ & $\alpha$ & $t$ & $N_{n}(\mathrm{Ag})$ & $\%$ & $N_{n}(\mathrm{Cu})$ & $\%$ \\
\hline 0.00 & 0.2 & 1.0 & 1.780 & - & - & - \\
0.01 & 0.2 & 1.0 & 1.857 & 4.325 & 1.852 & 4.044 \\
0.02 & 0.2 & 1.0 & 1.936 & 8.764 & 1.926 & 8.202 \\
0.04 & 0.2 & 1.0 & 2.202 & 24.820 & 2.081 & 16.910 \\
\hline
\end{tabular}

TABLE. 4: Distinction in the Sherwood number (rate of mass transfer).

\begin{tabular}{lcccc}
\hline$\alpha$ & $t$ & Sc & $\varphi$ & $S_{n}$ \\
\hline 0.2 & 1.0 & 5.0 & 0.01 & 1.124 \\
$\mathbf{0 . 3}$ & 1.0 & 5.0 & 0.01 & 1.091 \\
0.2 & 1.0 & $\mathbf{1 0}$ & 0.01 & 1.885 \\
0.2 & 1.0 & 5.0 & $\mathbf{0 . 0 2}$ & 1.105 \\
\hline
\end{tabular}

discussions, the following key observations have been carried out from this work:

(i) The fractional parameter $\alpha$ delivers more than one line as associated with the classical model. This influence describes the memory effects which is not possible to elaborate by the classical model.

(ii) For the solution, the new transformation is more reliable. It is very simple to solve the fractional model by using these transformations.

(iii) The velocity of the fluid rises by rising the value of $\mathrm{Gr}, \mathrm{Gm}$, and $\lambda_{1}$.

(iv) The fluid velocity drops by rising the value of Sc, $M, \lambda_{2}$, and $\varphi$.

(v) It is interesting to note that the heat transfer rate of engine oil is enhanced by $24.820 \%$ for $\mathrm{Ag}$ nanoparticles and $16.910 \%$ for $\mathrm{Cu}$ nanoparticles.

\section{Data Availability}

The data used to support the findings of this study are included within the article.

\section{Conflicts of Interest}

The authors declare that they have no conflicts of interest. 


\section{References}

[1] G. Kunti, A. Bhattacharya, and S. Chakraborty, "Analysis of micromixing of non-newtonian fluids driven by alternating current electrothermal flow," Journal of Non-Newtonian Fluid Mechanics, vol. 247, pp. 123-131, 2017.

[2] M. Hemmat Esfe, R. Karimpour, A. A. Abbasian Arani, and J. Shahram, "Experimental investigation on non-newtonian behavior of $\mathrm{Al}_{2} \mathrm{O}_{3}$-MWCNT/5W50 hybrid nano-lubricant affected by alterations of temperature, concentration and shear rate for engine applications," International Communications in Heat and Mass Transfer, vol. 82, pp. 97-102, 2017.

[3] S. N. Li, H. N. Zhang, X. B. Li et al., "Numerical study on the heat transfer performance of non-newtonian fluid flow in a manifold microchannel heat sink," Applied Thermal Engineering, vol. 115, pp. 1213-1225, 2017.

[4] R. P. Chhabra and J. F. Richardson, Non-Newtonian Flow and Applied Rheology: Engineering Applications, ButterworthHeinemann, Oxford, England, 2011.

[5] T. Hayat, S. A. Shehzad, M. Qasim, and S. Obaidat, "Radiative flow of Jeffery fluid in a porous medium with power law heat flux and heat source," Nuclear Engineering and Design, vol. 243, pp. 15-19, 2012.

[6] M. Turkyilmazoglu and I. Pop, "Exact analytical solutions for the flow and heat transfer near the stagnation point on a stretching/shrinking sheet in a Jeffrey fluid," International Journal of Heat and Mass Transfer, vol. 57, no. 1, pp. 82-88, 2013.

[7] R. Ellahi and F. Hussain, "Simultaneous effects of MHD and partial slip on peristaltic flow of Jeffery fluid in a rectangular duct," Journal of Magnetism and Magnetic Materials, vol. 393, pp. 284-292, 2015.

[8] F. Mabood, M. Imtiaz, and T. Hayat, "Features of CattaneoChristov heat flux model for stagnation point flow of a Jeffrey fluid impinging over a stretching sheet: A numerical study," Heat Transfer, vol. 49, no. 5, pp. 2706-2716, 2020.

[9] M. Qasim, "Heat and mass transfer in a Jeffrey fluid over a stretching sheet with heat source/sink," Alexandria Engineering Journal, vol. 52, no. 4, pp. 571-575, 2013.

[10] S. Siddiqa, N. Begum, M. A. Hossain, and R. S. R. Gorla, "Natural convection flow of a two-phase dusty non-newtonian fluid along a vertical surface," International Journal of Heat and Mass Transfer, vol. 113, pp. 482-489, 2017.

[11] S. U. Choi and J. A. Eastman, Enhancing Thermal Conductivity of Fluids with Nanoparticles (No. ANL/MSD/CP-84938; CONF-951135-29), Argonne National Laboratory, Lemont, IL, USA, 1995.

[12] F. Ali, S. Murtaza, I. Khan, N. A. Sheikh, and K. S. Nisar, "Atangana-Baleanu fractional model for the flow of Jeffrey nanofluid with diffusion-thermo effects: Applications in engine oil," Advances in Difference Equations, vol. 2019, no. 1, p. 346, 2019.

[13] M. Kole and T. K. Dey, "Effect of aggregation on the viscosity of copper oxide-gear oil nanofluids," International Journal of Thermal Sciences, vol. 50, no. 9, pp. 1741-1747, 2011.

[14] W. Tesfai, P. Singh, Y. Shatilla, M. Z. Iqbal, and A. A. Abdala, "Rheology and microstructure of dilute graphene oxide suspension," Journal of Nanoparticle Research, vol. 15, no. 10, p. $1989,2013$.

[15] M. Sheikholeslami and H. B. Rokni, "Simulation of nanofluid heat transfer in presence of magnetic field: A review," International Journal of Heat and Mass Transfer, vol. 115, pp. 1203-1233, 2017.
[16] Z. Haddad, E. Abu-Nada, H. F. Oztop, and A. Mataoui, "Natural convection in nanofluids: Are the thermophoresis and Brownian motion effects significant in nanofluid heat transfer enhancement?" International Journal of Thermal Sciences, vol. 57, pp. 152-162, 2012.

[17] K. Parekh and H. S. Lee, "Magnetic field induced enhancement in thermal conductivity of magnetite nanofluid," Journal of Applied Physics, vol. 107, no. 9, Article ID 09A310, 2010.

[18] S. Dinarvand, R. Hosseini, and I. Pop, "Axisymmetric mixed convective stagnation-point flow of a nanofluid over a vertical permeable cylinder by Tiwari-Das nanofluid model," Powder Technology, vol. 311, pp. 147-156, 2017.

[19] S. T. Mohyud-Din, Z. A. Zaidi, U. Khan, and N. Ahmed, "On heat and mass transfer analysis for the flow of a nanofluid between rotating parallel plates," Aerospace Science and Technology, vol. 46, pp. 514-522, 2015.

[20] P. Loganathan, P. Nirmal Chand, and P. Ganesan, "Radiation effects on an unsteady natural convective flow of a nanofluid past an infinite vertical plate," Nano, vol. 08, no. 01, Article ID 1350001, 2013.

[21] S. Ferrouillat, A. Bontemps, J. P. Ribeiro, J. A. Gruss, and O. Soriano, "Hydraulic and heat transfer study of $\mathrm{SiO}_{2} /$ water nanofluids in horizontal tubes with imposed wall temperature boundary conditions," International Journal of Heat and Fluid Flow, vol. 32, no. 2, pp. 424-439, 2011.

[22] E. T. Bell, Mathematics, Queen and Servant of Science, Oxford Press, England, UK, 1951.

[23] M. Lazarevic, M. R. Rapaic, T. B. Sekara et al., "Advanced topics on applications of fractional calculus on control problems," System Stability and Modeling, vol. 43, no. 4, pp. 202-215, 2014.

[24] T. F. Nonnenmacher and R. Metzler, "On the RiemannLiouville fractional calculus and some recent applications," Fractals, vol. 03, no. 03, pp. 557-566, 1995.

[25] M. Caputo, "Linear models of dissipation whose Q is almost frequency independent-II," Geophysical Journal International, vol. 13, no. 5, pp. 529-539, 1967.

[26] M. Caputo and M. Fabrizio, "A new definition of fractional derivative without a singular kernel," Progress in Fractional Differentiation and Applications, vol. 1, no. 2, pp. 1-13, 2015.

[27] A. Atangana and I. Koca, "Chaos in a simple nonlinear system with Atangana-Baleanu derivatives with fractional order," Chaos, Solitons \& Fractals, vol. 89, pp. 447-454, 2016.

[28] R. Metzler, W. Schick, H. G. Kilian, and T. F. Nonnenmacher, "Relaxation in filled polymers: A fractional calculus approach," The Journal of Chemical Physics, vol. 103, no. 16, pp. 7180-7186, 1995.

[29] C. Ionescu, A. Lopes, D. Copot, J. A. T. Machado, and J. H. T. Bates, "The role of fractional calculus in modeling biological phenomena: A review," Communications in Nonlinear Science and Numerical Simulation, vol. 51, pp. 141-159, 2017.

[30] Q. Al-Mdallal, K. A. Abro, and I. Khan, "Analytical solutions of fractional walter's B fluid with applications," Complexity, vol. 2018, Article ID 8131329, 10 pages, 2018.

[31] S. Alizadeh, D. Baleanu, and S. Rezapour, "Analyzing transient response of the parallel RCL circuit by using the Caputo-Fabrizio fractional derivative," Advances in Difference Equations, vol. 2020, no. 1, p. 55, 2020.

[32] A. Atangana and R. T. Alqahtani, "Numerical approximation of the space-time Caputo-Fabrizio fractional derivative and application to groundwater pollution equation," Advances in Difference Equations, vol. 2016, no. 1, pp. 1-13, 2016. 
[33] M. A. Dokuyucu, E. Celik, H. Bulut, and H. M. Baskonus, "Cancer treatment model with the Caputo-Fabrizio fractional derivative," The European Physical Journal Plus, vol. 133, no. 3, pp. 1-6, 2018.

[34] I. Ahmed, P. Kumam, T. Abdeljawad et al., "Existence and uniqueness results for $\varphi$-Caputo implicit fractional pantograph differential equation with generalized anti-periodic boundary condition," Advances in Difference Equations, vol. 2020, no. 1, pp. 1-19, 2020.

[35] E. F. Doungmo Goufo, "Application of the Caputo-Fabrizio fractional derivative without singular kernel to Korteweg-de vries-burgers equation," Mathematical Modelling and Analysis, vol. 21, no. 2, pp. 188-198, 2016.

[36] J. Hristov, "Transient heat diffusion with a non-singular fading memory: from the Cattaneo constitutive equation with Jeffrey's kernel to the Caputo-Fabrizio time-fractional derivative," Thermal Science, vol. 20, no. 2, pp. 757-762, 2016.

[37] I. Koca and A. Atangana, "Solutions of Cattaneo-Hristov model of elastic heat diffusion with Caputo-Fabrizio and Atangana-Baleanu fractional derivatives," Thermal Science, vol. 21, no. 6, pp. 2299-2305, 2017.

[38] C. RamReddy and P. Naveen, "Analysis of activation energy and thermal radiation on convective flow of a power-law fluid under convective heating and chemical reaction," Heat Transfer-Asian Research, vol. 48, no. 6, pp. 2122-2154, 2019.

[39] A. A. Farooq, Z. Shah, P. Kumam, E. O. Alzahrani, M. Shutaywi, and T. Anwar, "Darcy-Boussinesq model of cilia-assisted transport of a non-newtonian magneto-biofluid with chemical reactions," Applied Sciences, vol. 10, no. 3, p. 1137, 2020.

[40] S. Chakraborty, I. Sarkar, A. Ashok, I. Sengupta, S. K. Pal, and S. Chakraborty, "Thermo-physical properties of $\mathrm{Cu}-\mathrm{Zn}-\mathrm{Al}$ LDH nanofluid and its application in spray cooling," Applied Thermal Engineering, vol. 141, pp. 339-351, 2018. 